\title{
A glutathione-based system for defense against carbonyl stress in Haemophilus influenzae
}

\author{
Stephen P Kidd ${ }^{1 *}$, Donald Jiang ${ }^{1}$, Alexandra Tikhomirova ${ }^{1}$, Michael P Jennings ${ }^{2}$ and Alastair G McEwan ${ }^{3}$
}

\begin{abstract}
Background: adhC from Haemophilus influenzae encodes a glutathione-dependent alcohol dehydrogenase that has previously been shown to be required for protection against killing by $S$-nitrosoglutathione (GSNO). This group of enzymes is known in other systems to be able to utilize substrates that form adducts with glutathione, such as aldehydes.

Results: Here, we show that expression of adhC is maximally induced under conditions of high oxygen tension as well as specifically with glucose as a carbon source. adhC could also be induced in response to formaldehyde but not GSNO. An adhC mutant was more susceptible than wild-type Haemophilus influenzae Rd KW20 to killing by various short chain aliphatic aldehydes, all of which can be generated endogenously during cell metabolism but are also produced by the host as part of the innate immune response.
\end{abstract}

Conclusions: These results indicate that AdhC plays a role in defense against endogenously generated reactive carbonyl electrophiles in Haemophilus influenzae and may also play a role in defense against the host innate immune system.

Keywords: Stress response, H. influenzae, Reactive aldehydes

\section{Background}

Haemophilus influenzae is a $\gamma$-Proteobacterium adapted to the human host. It exists as a commensal in up to $80 \%$ of the healthy population. It survives in the nasopharnyx, and can spread to other sites within the body and cause disease [1]. $H$. influenzae requires a number of exogenous cofactors for growth including cysteine for the production of glutathione (GSH) [2]. In addition to its role in defence against oxidative stress [2,3] GSH forms adducts with toxic electrophilic molecules. Glutathionedependent alcohol dehydrogenase (AdhC) catalyses the $\mathrm{NAD}^{+}$-dependent oxidation of a GSH-formaldehyde adduct $[4,5]$. Expression of $a d h C$ in a variety of bacteria is associated with defense against formaldehyde stress and is correspondingly regulated in the response to the presence of formaldehyde [6]. It is also established that AdhC catalyses the NADH-dependent reduction of $S$-nitrosoglutathione (GSNO), a molecule generated during the conditions of

\footnotetext{
* Correspondence: stephen.kidd@adelaide.edu.au

${ }^{1}$ Research Centre for Infectious Disease, School of Molecular and Biomedical Science, The University of Adelaide, North Terrace Campus, Adelaide 5005, Australia

Full list of author information is available at the end of the article
}

nitrosative stress that occurs in human cells in response to invading pathogens such as $H$. influenzae. Unlike other aldehyde dehydrogenase enzymes AdhC cannot use ethanol or formaldehyde directly, but uses the adducts which spontaneously form with GSH (hence the nomenclature, GSH-dependent formaldehyde dehydrogenase) [7]. AdhC from different sources is known to catalyse the concurrent oxidation of formaldehyde and reduction of GSNO $[8,9]$. We have previously observed that AdhC of $H$. influenzae does function in GSNO metabolism [10].

H. influenzae does not use methanol as a carbon source (the by-product of which is formaldehyde) and cannot assimilate formaldehyde. Therefore, a source of formaldehyde substrate for AdhC from the host environment is not obvious; however, bacteria do encounter a variety of aldehydes. Neutrophils use myeloperoxidase to produce glycoaldehyde and acrolein at sites of inflammation while short chain sugar aldehydes such as glyceraldehyde 3-P and erythrose 4-P are produced endogenously as intermediates of the bacterial metabolism of sugars [11]. All these short chain aldose sugars mentioned can undergo auto-oxidation to more toxic dicarbonyl species [12]. In this paper we report the effect of reactive carbonyl species 
on growth of $H$. influenzae. This provides a new insight into the physiological role of AdhC in non-methylotrophic bacteria.

\section{Methods}

Bacterial strains and growth conditions

$H$. influenzae strains were cultured on Brain heart infusion (BHI) medium or chemically defined media (CDM). BHI was prepared with $3.7 \%$ (wt/vol) BHI Powder (Oxoid). For solid medium, 1.5\% (wt/vol) agar powder was added. Medium was sterilized by autoclaving at $121^{\circ} \mathrm{C}$ for $20 \mathrm{~min}$. Levinthal blood (10\% [wt/vol]) was added for solid medium. BHI broth required NAD $(2 \mu \mathrm{g} / \mathrm{ml})$ and $10 \mu \mathrm{g} / \mathrm{ml}$ hemin solution $(0.1 \%$ [wt/vol] hemin, $0.1 \%$ [wt/vol] L-histidine, $4 \%$ [vol/vol] triethanolamine). Solutions for media were sterilized individually, either by filter sterilizing or by autoclaving. The solutions were mixed under sterile conditions. CDM was prepared mostly as described by Coleman et al. [13]. The exception to this protocol is the use of RPMI 1640 without glucose (Invitrogen) and the addition of $0.4 \%$ of the appropriate sugar or carbon source. In standard procedures the final $\mathrm{pH}$ of CDM was adjusted to 7.56 by $\mathrm{NaHCO}_{3}$. CDM was sterilized by filter sterilization through a $0.22-\mu \mathrm{m}$ filter.

\section{Reverse transcriptase PCR}

RNA was extracted from $H$. influenzae Rd KW20 at the time points $3 \mathrm{~h}, 5.5 \mathrm{~h}$ and $8 \mathrm{~h}$ during growth cycle by using a QIAGEN RNeasy minikit (QIAGEN). RNA was quantified using an $\mathrm{A}_{260}$ reading and then checked for DNA contamination by PCR; no product was detected. RNA was further treated to remove any residual DNA by using Promega DNase (Promega). The reverse transcriptase (RT) reaction was performed using a QIAGEN Omniscript reverse transcriptase kit. The products of this reaction were used in a multiplex PCR with primers for the $16 \mathrm{~S}$ rRNA gene:

16SFOR: 5'-AGTCCACGCCCTAAACGATGT-3' and 16SREV: 5'-TACTCCCCAGGCGGTCAAT-3'; and primers from $e s t D$ to $a d h C$ :

Est1: 5'-CCCAAGGCTGCTCGGTC-3' and Adh1, 5'-TTCAACGCGTCCGTTCCAA-3'.

PCR was carried out with New England Biolabs Taq polymerase using an initial $96^{\circ} \mathrm{C}$ for $10 \mathrm{~min}$ followed by 30 cycles of $96^{\circ} \mathrm{C}$ for $45 \mathrm{~s}, 54^{\circ} \mathrm{C}$ for $45 \mathrm{~s}$, and $72^{\circ} \mathrm{C}$ for $30 \mathrm{~s}$ and a final elongation step of $72^{\circ} \mathrm{C}$ for $10 \mathrm{~min}$.

\section{Growth assays}

Cells were cultured in rich media (BHI, Oxoid UK) or chemically defined media (CDM). Unless otherwise stated, analysis of the growth of $H$. influenzae strains was carried out using CDM. For rich media cells were grown on BHI medium supplemented with NAD $(2 \mu \mathrm{g} / \mathrm{ml})$ and $10 \mu \mathrm{g} / \mathrm{ml}$ hemin solution. Overnight growth cultures were inoculated into $5 \mathrm{ml}$ of media and grown until log phase prior to the assay. The $\mathrm{OD}_{600}$ of the cultures were measured (Hitachi U-3000 spectrophotometer) to standardize the amount of cells inoculated. Routinely $1 \mathrm{ml}$ was inoculated into $50 \mathrm{ml}$ of CDM in a 250-ml conical flask. For analysis of the effects of oxygen supply to the cells, cultures were grown in $250 \mathrm{ml}$ conical flasks with $25 \mathrm{ml}$, $75 \mathrm{ml}$ and $150 \mathrm{ml}$ medium. This has been previously used and shown to provide the oxygen transfer coefficents (kLa) values of $87.4 \mathrm{~h}^{-1}$ (high), $27.8 \mathrm{~h}^{-1}$ (medium) and $11.5 \mathrm{~h}^{-1}$ (low) respectively $[14,15]$.

Different specific concentrations of stress agent were added to the medium. Cultures were incubated aerobically at $37^{\circ} \mathrm{C}$ with shaking at $190 \mathrm{rpm}$. $\mathrm{OD}_{600}$ measurements were taken at different time points for $10 \mathrm{~h}$. The assays were done in triplicate. Assay results were represented as growth curves over this period or, for clarity for the large set of clinical isolates, as percentages of survival at this time point.

\section{GSNO reductase enzyme assays}

NADH-dependent GSNO reductase activity was measured as previously described [10]. Fresh overnight cultures of $H$. influenzae were inoculated into $100 \mathrm{ml}$ of $\mathrm{CDM}$ in $500 \mathrm{ml}$ conical flasks and grown aerobically at $37^{\circ} \mathrm{C}$ with shaking at $190 \mathrm{rpm}$ until an $\mathrm{OD}_{600}$ measurement between 0.4 and 0.6 was obtained. The cells were harvested $\left(5,000 \times \mathrm{g}\right.$ at $4^{\circ} \mathrm{C}$ for $\left.10 \mathrm{~min}\right)$ and washed twice with $0.1 \mathrm{M}$ phosphate buffer ( $\mathrm{pH}$ 7.0) before resuspending in $2 \mathrm{ml}$ of phosphate buffer. The suspension was frozen at $-80^{\circ} \mathrm{C}$, thawed at room temperature, given a brief vortexing, and frozen again at $-80^{\circ} \mathrm{C}$. This freezethaw process was performed four more times before the cells were centrifuged at $13,000 \times \mathrm{g}$ at $4^{\circ} \mathrm{C}$ for $15 \mathrm{~min}$. The final supernatant (cell extract) was used for assays. The total protein concentration of the supernatant was determined spectrophotometrically using the formula protein $(\mathrm{mg} / \mathrm{ml})\left(1.55 \times \mathrm{A}_{280}\right)-\left(0.76 \times \mathrm{A}_{260}\right)^{19}$. GSNO reductase activity was expressed as $\mu \mathrm{mol}$ of NADH oxidized per minute per mg of total protein. The assays were done in triplicate.

\section{Results}

AdhC is expressed under aerobic conditions and required for aerobic growth in $\mathrm{H}$. influenzae

We have previously observed that an adhC mutant of H. influenzae $\mathrm{Rd}$ KW20 appeared to have a reduced growth under aerobic conditions compared to its wildtype strain [10]. To further characterize this altered phenotype and determine its direct link to aerobic growth pathways and oxygen, we performed various growth assays using established parameters for low, 
medium and high levels of aeration to correlate to oxygen levels. We also used rich media and chemically defined media (providing only glucose as the carbon source) (Figure 1A and 1B). At high oxygen levels and in
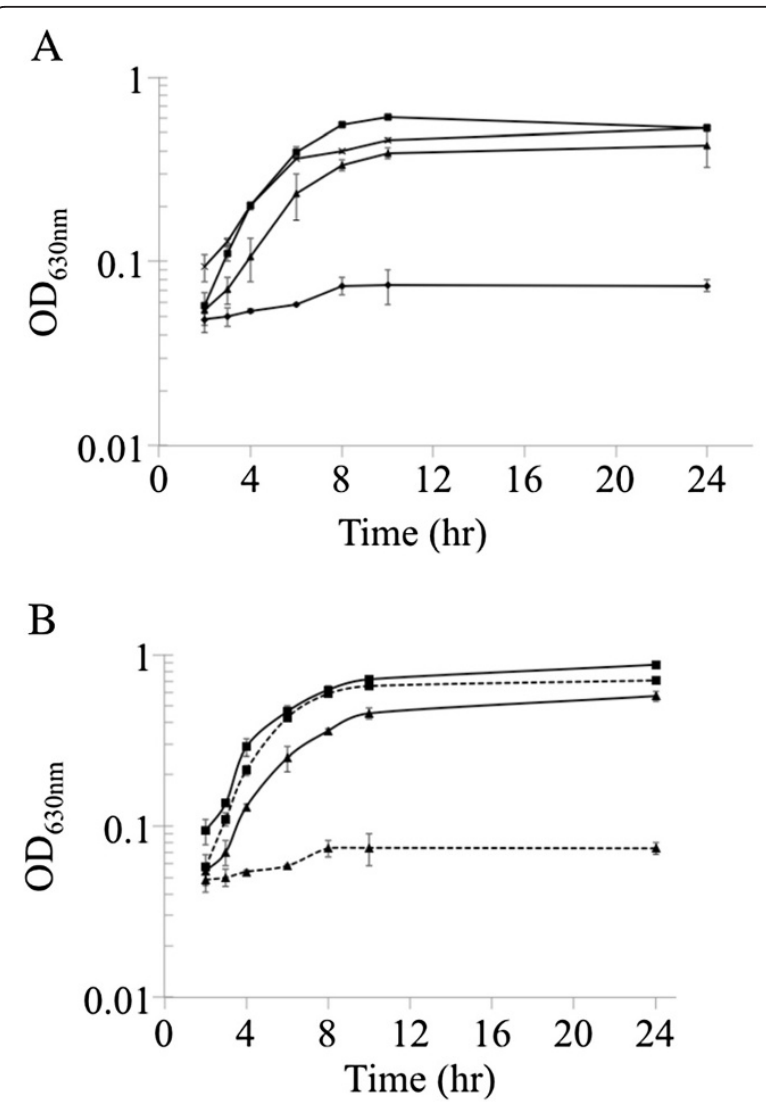

C

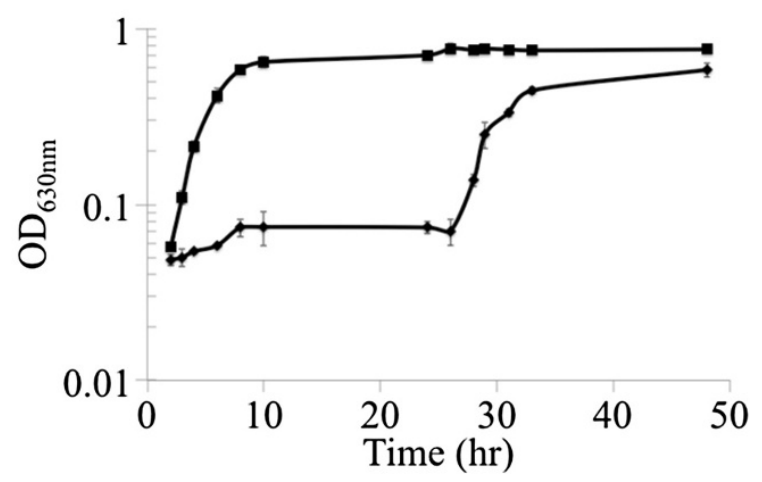

Figure $1 \mathrm{AdhC}$ in $\mathrm{H}$. influenzae is required for growth with glucose at high oxygen. (A) The Rd KW20 wild type and adhC mutant were grown in CDM at low oxygen tension ( $x$, RdKW20 and $\boldsymbol{\Delta}$, adhC mutant) and then high oxygen tension ( $\square$, RdKW20 and adh C mutant). (B) As a comparison the Rd KW20 was grown in BHI (-) and $\mathrm{CDM}(\mathbf{-})$ and the $a d h C$ mutant was grown in $\mathrm{BHI}(\mathbf{\Delta})$ and $\mathrm{CDM}$ ( $\Delta$ with dotted lines). (C) Rd KW20 ( $\bullet$ ) and adhC mutant ( $)$ were then grown with high oxygen until $24 \mathrm{hr}$ when the oxygen tension was changed to low oxygen.
CDM the adhC mutant did not grow. Both wild type and $a d h C$ mutant cells were then grown at high oxygen for $24 \mathrm{~h}$ before being directly transferred to low oxygen conditions for a further $20 \mathrm{~h}$ (Figure 1C). Upon the switch in oxygen tension the $a d h C$ mutant cells grew.

To assess whether AdhC was being expressed under these aerobic conditions in the wild type cells we firstly monitored AdhC activity during the growth cycle. The cells were assayed for AdhC activity (by assay of GSNO reductase activity), at different time points through the growth cycle. Figure 2A shows that AdhC activity increases during exponential phase, and then decreases in late exponential and stationary phase. RNA was also extracted from $H$. influenzae wild-type strain at early, mid and late log phase and RT-PCR was performed using $16 \mathrm{~S}$ and adhC-estD primers (Figure 2B). We also investigated the effect of differences in oxygen tension on AdhC expression by growing cultures in low, medium and high oxygen levels; Figure $2 \mathrm{C}$ shows that AdhC activity was highest in cells grown at highest oxygen tension and activity decreased as oxygen tension in the culture decreased. Taken together these results indicated that adhC expression in $H$. influenzae is highest under aerobic conditions and this is associated with glucose metabolism.

\section{AdhC is required for defense against reactive aldehydes}

To determine whether AdhC had a role in protection against the reactive aldehydes known to be relevant and toxic during aerobic growth, we grew wild-type (Rd KW20) and its isogenic $a d h C$ mutant in the presence of some of these compounds and measured the end point of growth $\left(\mathrm{OD}_{600}\right)$, growth of any culture did not continue beyond the $18 \mathrm{hr}$ point. Figure 3 shows that the adhC strain was more sensitive than wild-type to methylglyoxal; $2 \mathrm{mM}$ methylglyoxyl completely inhibited the growth of the $a d h C$ mutant but had very little effect on growth of wild-type $H$. influenzae Rd KW20. Glyceraldehyde, glycolaldehyde and glyoxal also inhibited growth of the adhC mutant compared to wild-type H. influenzae Rd KW20. The overall growth profiles (lag phase and growth rates) were equally reduced in the adhC mutant compared to wild type. It has been demonstrated that the toxicity of short chain sugars, such as glyceraldehyde and glycolaldehyde, arises from the oxidation of their ene-diol tautomeric form which results in the formation of highly toxic dicarbonyl species [12]. If failure to protect against toxic dicarbonyl species underpinned the increased toxicity of reactive aldehydes towards the $a d h C$ mutant, then it ought to be possible to rescue such mutants using $1 \mathrm{mM} \mathrm{1,2-}$ diaminobenzene $(\mathrm{DAB})$ a compound that quenches the toxicity of dicarbonyl species. The addition of DAB did partially restore the growth of the $a d h C$ mutant in the 


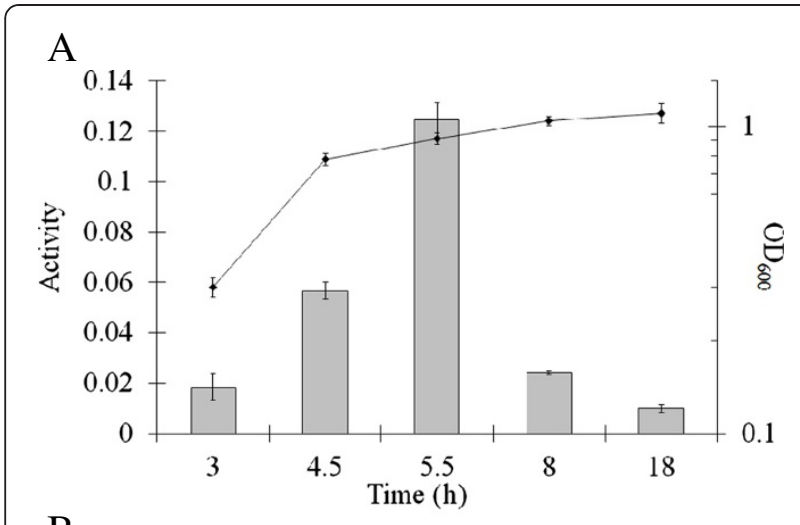

B

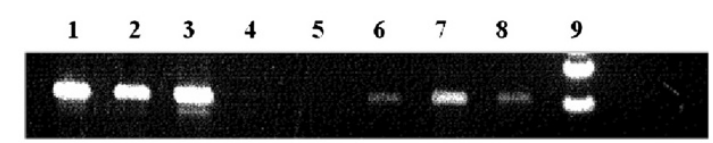

C

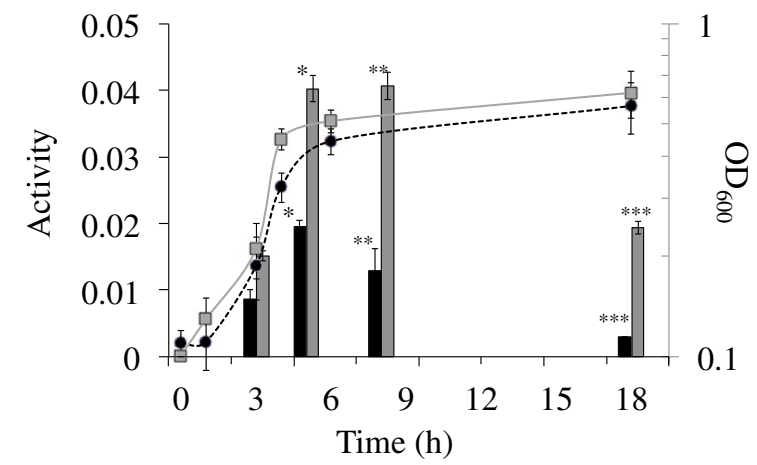

Figure $\mathbf{2}$ Change in AdhC specific activity during growth of H. influenzae. (A) Samples were taken and assayed for AdhC enzyme activity from early log phase (3 hr), mid-log phase ( $4.5 \mathrm{~h}$ ), log phase $(5.5 \mathrm{~h}$ ) late log phase $(8 \mathrm{~h})$ and stationary phase (18 h). (B) RT-PCR for the 16SrDNA (lanes 1-4) and adhC-estD (lanes 5-8) using RNA from the time points $3 \mathrm{~h}$ (lanes 1 and 6 ), $5.5 \mathrm{~h}$ (lanes 2 and 7) and $8 \mathrm{~h}$ (lanes 3 and 8). Lanes 4 and 5 are representative negative controls. Lane 9 is the ladder. (C) At time points throughout the $\mathrm{H}$. influenzae growth phase AdhC specific activity was measured from cells grown with different oxygen tensions (low tension are the black bar and high oxygen tension are the grey bars). The enzyme activity is presented as change in $\mathrm{NADH}$ consumed per minute per $\mathrm{mg}$ total protein. Y-error bars indicate $+/-1$ standard deviation of the mean. Units are $\mu$ mol NADH oxidized $\mathrm{min}^{-1} \mathrm{mg}$ protein ${ }^{-1}$. The growth curves are indicated by the $\mathrm{OD}_{600}$ of cells grown at low oxygen levels (black circle) and high oxygen levels (gray box). $\left({ }^{*} P<0.001,{ }^{* *} P<0.002,{ }^{* * *} P<0.0005\right)$.

presence of glycolaldehyde (Table 1). Consistent with this, under conditions of low oxygen where the toxic effect of these molecules is reduced, the susceptibility of the $a d h C$ mutant to these aldehydes is reduced (Figure 3). Given that previous studies on bacterial AdhC enzymes have focussed on its role in formaldehyde detoxification, we also assayed for formaldehyde sensitivity in the $H$. influenzae adhC mutant. The adhC mutant was slightly more sensitive than wild type to formaldehyde under high oxygen conditions when cultured in CDM, but was not at all under low conditions (Figure 3).

\section{AdhC is induced by formaldehyde but not by GSNO}

To determine whether the NmlR system, which controls AdhC expression, responded to nitrosative stress we investigated the effect of GSNO on AdhC activity. There was no change in AdhC activity upon addition of GSNO (the Units of activity remained at the same level as none added; $0.02 \pm 0.005 \mu \mathrm{mol}$ of NADH oxidized per minute per $\mathrm{mg}$ of total protein), suggesting that $\mathrm{NmlR}_{\mathrm{HI}}$ in $H$. influenzae does not respond to nitrosative stress (Figure 4). As previously investigated, Escherichia coli and $H$. influenzae cells grown with formaldehyde had higher AdhC activity [16]; we tested a range of reactive aldehydes to ascertain whether they could induce $a d h C$ expression in $H$. influenzae. Figure 4 shows that addition of formaldehyde to $H$. influenzae caused a 5-fold rise in AdhC activity 5 minutes after its addition. AdhC activity was not induced by methylglyoxyl and glycolaldehyde under the same conditions (in both cases the Units of activity remained at the same level as with no chemical added; $0.02 \pm 0.005 \mu \mathrm{mol}$ of $\mathrm{NADH}$ oxidized per minute per mg of total protein).

\section{Discussion}

The expression of $a d h C$ is regulated by the MerR family transcription factor $\mathrm{NmlR}_{\mathrm{HI}}[10]$. Regulators of this family generally function as both weak repressors, and as activators when in the presence of their cognate stress effector. We have previously reported that expression of GSNO reductase activity in $H$. influenzae requires both $a d h C$, the structural gene encoding the enzyme activity, as well as its regulator $n m l R_{\mathrm{HI}}$ under growth conditions with no exogenous stress. Mutant strains of $H$. influen$z a e$ in which the $a d h C$ or $n m l R_{\mathrm{HI}}$ genes have been inactivated do not express detectable GSNO reductase activity [10]. A reasonable conclusion was that under these conditions $\mathrm{NmlR}_{\mathrm{HI}}$ is in its activator conformation and therefore endogenously generated molecules are the cognate "stress" for which it responds. Attempts to identify the cognate ligand or the environmental stimuli, which acts to switch $\mathrm{NmlR}_{\mathrm{HI}}$, to an activator form have been unsuccessful.

In mammalian systems AdhC functions in detoxification of a range of reactive aldehyde species as well as in defense against GSNO. Our results suggest that there may be a similar role for AdhC in $H$. influenzae. Glycoaldehyde is produced from serine by the action of myeloperoxidase [17]. This is one of several types of reactive aldehydes that are produced by activated neutrophils at sites of inflammation. The toxicity of glycoaldehyde arises from the oxidation of its ene-diol tautomer 


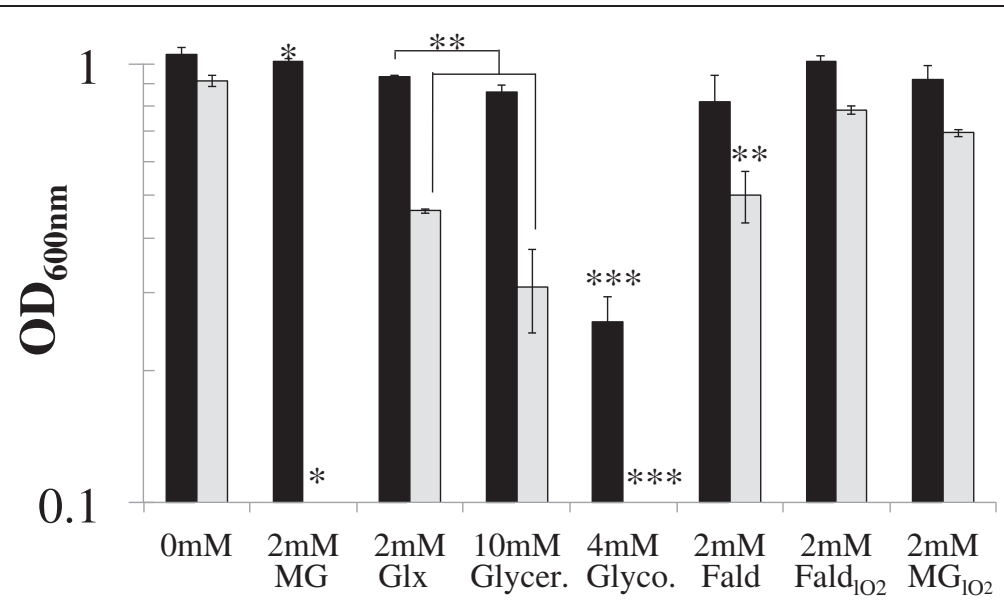

Figure 3 Sensitivity of $\boldsymbol{H}$. influenzae adhC strain to reactive aldehydes. Wild type (Rd KW20; black bars) and the adhC mutant (grey bars) strains were grown in $\mathrm{BH}$ media in the presence of increasing concentrations of particular reactive aldehydes with medium levels of oxygen ( $50 \mathrm{ml}$ culture in $250 \mathrm{ml}$ flask). The ability to resist the toxicity of these chemicals was measured by an $\mathrm{OD}_{600}$ reading after $18 \mathrm{~h}$ of growth. $\left({ }^{*} P<0.0001,{ }^{* *} P<0.005,{ }^{* * *} P<0.0001\right)$. MG: methylglyoxal, Glx: glyoxal, Glycer: glyceraldehyde, Glyco: glycolaldehyde, Fald: formaldehyde, Fald $\mathrm{IO}_{\mathrm{O}}$ : formaldehyde with low oxygen, $\mathrm{MG}_{\mathrm{OO} 2}$, methylglyoxal with low oxygen.

to form a highly reactive $\alpha, \beta$-dicarbonyl species. This reaction requires oxygen or superoxide, consistent with AdhC activity being highest with increased oxygen levels and during the highest periods of metabolic reactions. Our observations are also consistent with previous in silico analyses analysis of gene expression in $H$. influen$z a e$ which showed that adhC expression was highest under aerobic conditions during which glucose would be metabolised mainly via the pentose phosphate pathway $[18,19]$. Our data is consistent with these results as AdhC was required for growth with glucose as the carbon source under high oxygen culture conditions (Figures 1 and 2). Glyceraldehyde 3-phosphate and erythrose 4phosphate are both intermediates in this pathway. It has been noted that the equilibrium constant for the aldolase reaction means that in glycolysis the concentration of glyceraldehyde 3-phosphate is kept very low. This may not be the case when the pentose phosphate pathway is the dominant glucose oxidation pathway that occurs under conditions of high oxygen tension $[18,19]$.

Recently, it is has been observed that an NmlR homologue in Bacillus subtilis (AdhR) activates gene

Table 1 The growth rates of Rd KW20 and adhC; with $2 \mathrm{mM}$ glycolaldehyde and $1 \mathrm{mM}$ 1,2-diaminobenzene (DAB)

\begin{tabular}{lc}
\hline Strains & Growth rate (doubling per hour) \\
\hline Rd KW20 & $1.10 \pm 0.14$ \\
Rd KW20 + glycolaldehyde & $0.80 \pm 0.37$ \\
Rd KW20 + glycol. + DAB & $1.47 \pm 0.35$ \\
adhC & $0.79 \pm 0.34$ \\
adhC + glycolaldehyde & $0.20 \pm 0.10$ \\
adhC + glycol. + DAB & $0.51 \pm 0.27$ \\
\hline
\end{tabular}

expression in response to methylglyoxyl and formaldehyde [20]. One cysteine (C54) was shown to be required for activation of gene expression and this led Antelmann and co-workers [20] to propose that Bacillus AdhR is activated by $S$-alkylation of this cysteine residue. AdhR contains a single conserved cysteine, as in the $\mathrm{NmlR}_{\mathrm{sp}}$ transcription factor from Streptococcus pneumoniae [21]. In $H$. influenzae we only observed induction of $a d h C$ by $\mathrm{NmlR}_{\mathrm{HI}}$ upon addition of formaldehyde. In contrast to the situation in B. subtilis and S. pneumoniae, $\mathrm{NmlR}_{\mathrm{HI}}$ possesses three conserved cysteine residues and is closely related to the NmlR regulators from Neisseria species [22]. Thus, there may be significant differences in the mechanism of the sensing of reactive carbonyl compounds by transcription factors of the NmlR family.

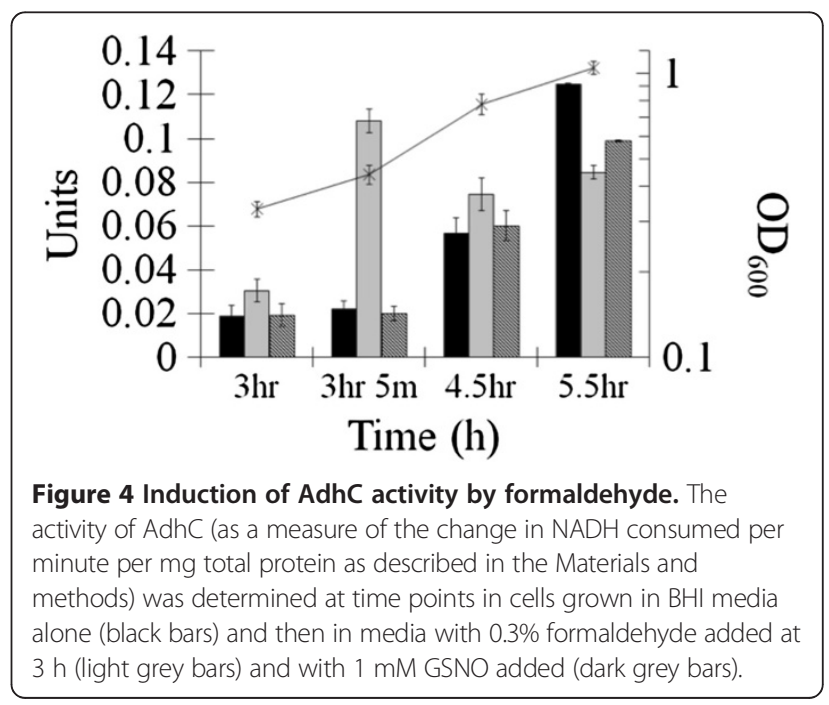




\section{Conclusions}

Uniquely, $H$. influenzae utilizes an AdhC enzyme for the concurrent roles of protection against an exogenous stress (GSNO) as well as the endogenously generated and harmful reactive aldehydes. AdhC is essential for $H$. influenzae growth under conditions of high oxygen and with glucose as the carbon source. This role is through the detoxification of different reactive carbonyl compounds.

\section{Competing interests}

The authors declare that they have no competing interests.

\section{Authors' contributions}

SPK helped in the design of the study, participated in the growth studies, the enzyme assays and the RT-PCR experiments and, helped draft the manuscript. DJ and AT participated in the growth studies. MPJ and AGM were part of the design and conception of the study and the analysis of the data and writing the manuscript. All authors read and approved the final manuscript.

\section{Acknowledgements}

We acknowledge support from program grants 284214 from the National Health and Medical Research Council of Australia to M. P. J. and A. G. M. and DP0986578 from the Australian Research Council to A. G. M.

\section{Author details}

${ }^{1}$ Research Centre for Infectious Disease, School of Molecular and Biomedical Science, The University of Adelaide, North Terrace Campus, Adelaide 5005, Australia. ${ }^{2}$ Institute for Glycomics, Griffith University (Gold Coast Campus), Parklands Drive, Southport, Queensland 4215, Australia. ${ }^{3}$ School of Chemistry and Molecular Biosciences, University of Queensland, BrisbaneQueensland 4072, Australia.

Received: 16 April 2012 Accepted: 25 July 2012

Published: 31 July 2012

\section{References}

1. Marrs CF, Krasan GP, McCrea KW, Clemans DL, Gilsdorf JR: Haemophlius influenzae - human specific bacteria. Front Biosci 2001, 6:e41-e60.

2. Vergauwen B, Pauwels F, Vaneechoutte M, Van Beeumen JJ: Exogenous glutathione completes the defense against oxidative stress in Haemophilus influenzae. J Bact 2003, 185:1572-1581.

3. Vergauwen B, Pauwels F, Van Beeumen JJ: Glutathione and catalase provide overlapping defenses for protection against repiration generated hydrogen peroxide in Haemophilus influenzae. J Bact 2003, 185:5555-5562.

4. Barber RD, Donohue TJ: Function of a glutathione-dependent formaldehyde dehydrogenase in Rhodobacter sphaeroides formaldehyde oxidation and assimilation. Biochem 1998, 37:530-537.

5. Hickman JW, Barber RD, Skaar EP, Donohue TJ: Link between the membrane bound pyridine nucleotide transhydrogenase and glutathione-dependent processes in Rhodobacter sphaeriodes. J Bact 2002, 184:400-409.

6. Hickman JW, Witthuhn VC Jr, Dominguez M, Donohue TJ: Positive and negative transcriptional regulators of glutathione-dependent formaldehyde metabolism. J Bact 2004, 186:7914-7925.

7. Staab C, Hellgren M, Höög JO: Medium- and short-chain dehydrogenase/ reductase gene and protein families. Cell Mol Life Sci 2008, 65:3950-3960.

8. Wu H, Romieu I, Sienra-Monge J-J, EsteladelRio-Navarro B, Anderson DM, Jenchura CA, Li H, Ramirez-Aguilar M, del Carmen Lara-Sanchez I, London SJ: Genetic variation in S-nitrosoglutathione reductase (GSNOR) and childhood asthma. J All Clin Imm 2007, 120:322-328.

9. Thompson CM, Grafstroum RC: Mechanistic considerations for formaldehyde-induced bronchoconstriction involving S-nitrosoglutathione reductase. J Tox Environl Health, Part A 2008, 71:244-248.

10. Kidd SP, Jiang D, Jennings MP, McEwan AG: A glutathione-dependent Alcohol Dehydrogenase (AdhC) is required for defense against nitrosative stress in Haemophilus influenzae. Infect Immun 2007, 75:4506-4513.
11. Anderson MM, Hazen SL, Hsu FF, Heinecke JW: Human neutrophils employ the myeloperoxidase-hydrogen peroxide-chloride system to convert hydroxy-amino acids into glycolaldehyde, 2-hydroxypropanal, and acrolein. A mechanism for the generation of highly reactive alphahydroxy and alpha,beta-unsaturated aldehydes by phagocytes at sites of inflammation. J Clin Invest 1997, 99:424-432.

12. Okado-Matsumoto A, Fridovich I: The role of alpha, beta-dicarbonyl compounds in the toxicity of short chain sugars. J Biol Chem 2000, 275:34853-34857.

13. Coleman HN, Daines DA, Jarisch J, Smith AL: Chemically defined media for growth of Haemophilus influenzae strains. J Clin Micro 2003, 41:4408-4410.

14. Cooper M, Tavankar GR, Williams HD: Regulation of expression of the cyanide-insensitive terminal oxidase in Pseudomonas aeruginosa. Microbio/ 2003, 149(5):1275-1284

15. Pirt SJ: Oxygen demand and supply. In Principles in Microbe and Cell Cultivation. Oxford: Blackwell; 1975:81-116.

16. Gutheil WG, Kasimoglu E, Nicholson PC: Induction of glutathionedependent formaldehyde dehydrogenase activity in Escherichia coli and Haemophilus influenzae. Biochem Biophysl Res Comm 1997, 238:693-696.

17. Anderson MM, Requena JR, Crowley JR, Thorpe SR, Heinecke JW: The myeloperoxidase system of human phagocytes generates NÎ $\mu$ (carboxymethyl)lysine on proteins: a mechanism for producing advanced glycation end products at sites of inflammation. J Clin Invest 1999, 104:103-113.

18. Edwards JS, Palsson BO: Systems Properties of the Haemophilus influenzae Rd Metabolic Genotype. J Biol Chem 1999, 274:17410-17416.

19. Raghunathan A, Price ND, Galperin MY, Makarova KS, Purvine S, Picone AF, Cherny T, Xie T, Reilly TJ, Munson R, et al: In Silico metabolic model and protein expression of Haemophilus influenzae Strain Rd KW20 in rich medium. OMICS: A J Inte Biol 2004, 8:25-41.

20. Huyen NTT, Eiamphungporn W, Mader U, Liebeke M, Lalk M, Hecker M, Helmann JD, Antelmann H: Genome-wide responses to carbonyl electrophiles in Bacillus subtilis: control of the thiol-dependent formaldehyde dehydrogenase AdhA and cysteine proteinase YraA by the MerR-family regulator YraB (AdhR). Mol Micro 2009, 71:876-894.

21. Stroeher UH, Kidd SP, Stafford SL, Jennings MP, Paton JC, McEwan AG: A pneumococcal MerR-like regulator and S-nitrosoglutathione reductase are required for systemic virulence. J Infect Dis 2007, 196:1820-1826.

22. Kidd SP, Potter AJ, Apicella MA, Jennings MP, McEwan AG: NmIR of Neisseria gonorrhoeae: a novel redox responsive transcription factor from the MerR family. Mol Micro 2005, 57:1676-1689.

doi:10.1186/1471-2180-12-159

Cite this article as: Kidd et al:: A glutathione-based system for defense against carbonyl stress in Haemophilus influenzae. BMC Microbiology 2012 $12: 159$

\section{Submit your next manuscript to BioMed Central and take full advantage of:}

- Convenient online submission

- Thorough peer review

- No space constraints or color figure charges

- Immediate publication on acceptance

- Inclusion in PubMed, CAS, Scopus and Google Scholar

- Research which is freely available for redistribution 